\title{
Pathological Influences of Twelve Months Vasectomy on the Reproductive Tissues in Rabbits
}

\author{
Xinggang Wang, Yuanfeng Zhang, Zonglin Chen, Xunbin Huang* \\ ${ }^{1}$ Family Planning Research Institute, Tongji Medical College, Huazhong University of Science and Technology, \\ Wuhan, China \\ Email: *huangxb@mails.tjmu.edu.cn
}

Received November 25, 2011; revised December 27, 2011; accepted January 10, 2011

\begin{abstract}
Objective-The effects of vasectomy on testes and related structures are disputable. The aim of this study was to determine whether the reproductive organs of rabbits were influenced after 12 months vasectomy. Study design —Bilateral vasectomy was performed on 10 male adult New Zealand Big Eared White Rabbits (another 10 rabbits set as sham operated group) and tissue samples were examined by light microscopy after 12 months treatment. The histologic alterations were observed and analyzed by stereology method. Cell apoptosis by Terminal Deoxynucleotidyl Transferase Mediated dUTP Nick End Labeling (TUNEL) assay were employed. Result-Bilateral vasectomy for 12 months affected the morphology of testis, epididymis and vas deferens tissues. Significant changes were noted in the histological stereology analysis. The apoptosis of the cells on the testis, epididymis and vas deferens increased compared with the sham operated group. Conclusion-Our findings suggest that the reproductive tissues of rabbits were influenced in a relatively longer term vasectomy.
\end{abstract}

Keywords: Vasectomy; Testis; Epididymis; Pathology; Rabbit

\section{Introduction}

Vasectomy was first used as a procedure for male contraception in 1899 [1]. Currently, about more than 40 million couples rely on it to prevent pregnancy over the world [2]. Traditionally, this operation is thought to be a convenience and high safety method. However, side effects such as the stasis of epididymis, painful nodules and sperm granuloma limit the acceptability of using this technique [3].

The effect of vasectomy on testis and related organs between species are disputable. Flickinger et al [4]. Reported that after vasectomized the rats, significant alterations of testis were observed. In the contrast of this finding, another report reported by McDonald found no changes in testis of rats [5]. There are also contradictory reports regarding the short-term and long-term effect.

At the same time, the requirements of vasectomy reversal increase every year [6], therefore it is mandatory to evaluate the function of the reproductive organs following vasectomy for long term using.

So, monitoring the progressive changes in the testis and related structures after long-term operation brook no delay. In order to evaluate these damages in the testis, epididymis and vas deferens at post-operation, the bilateral vasectomy and sham operation were carried out on 10 male rabbits respectively and the samples were obtained after 12 months. Attempts were also made to confirm the effects of vasectomy on cell apoptosis of these organs.

\section{Materials and Methods}

\subsection{Animal and Experimental Design}

Twenty normal male New Zealand Big Eared White Rabbits, aged 5 - 6 months, weighting 2.1 - $3.7 \mathrm{~kg}$, were obtained from the Experimental Animal Centre, Huazhong University of Science and Technology (HUST). The animals were allowed to acclimate to their new surroundings for at least 1 week. Then the rabbits were marked according to their weight and divided into two groups (vasectomy and sham operated groups) randomly. The animal experiment was approved by the Institutional Review Board at Huazhong University of Science and Technology.

\subsection{Vasectomy and Sham Operation}

Anesthesia was induced by intra-peritoneal injection of sodium pentobarbital at a dose of $30 \mathrm{mg} / \mathrm{kg}$ body weight. Under sterile conditions, a scrotal incision was made to expose the vas deferens and about $2-3 \mathrm{~cm}$ length of it was excised with the two ends ligated in the vasectomy 
group. In the sham operated group, however, the vas deferens was only exposed without cutting off. After operations, each animal was treated with $80,000 \mathrm{U}$ penicillin with intramuscular injection for three days and kept the wounds dry to avoid infection. The rabbits were raised as routine in the animal center for 12 months.

\subsection{Tissue Processing}

Twelve months after operation, organs removal was performed under anesthesia. Testes were immersed in Bouin fluid, and epididymis and the vas deferens were fixed with $10 \%$ formalin solution for 24 hours. Then the samples were dehydrated in ethanol and immersed in paraffin.

Testis, epididymis and vas deferens were cut into parallel slices about $10 \mu \mathrm{m}$ thickness. Some of the slices were stained with hematoxylin and eosin (H \& E) and then observed the changes under the light microscopy.

For the apoptosis study, the slices were processed under the manufacture's introduction (Roche Company, Switzerland). Slices were de-waxed and dehydrated. Then the specimens were digested with proteinase-K (20 $\mu \mathrm{g}$ in Tris/HCl) for $20 \mathrm{~min}$ and then dried in the air condition. The slices were fixed in $4 \%$ paraformaldehyde solution 5 min. After the slices were fixed, slices were washed with Phosphate Buffered Saline (PBS) and incubated in 0.3\% formaldehyde solution for $30 \mathrm{~min}$ at the room temperature, then washed with PBS again. Then incubated the slices with $50 \mu \mathrm{l}$ of TUNEL reaction mixture solution at $37^{\circ} \mathrm{C}$ in a wet box for $60 \mathrm{~min}$, and then washed with PBS three times. After the slides were dried, $50 \mu$ l of biotin labeled peroxidase (POD) was added and incubated for $30 \mathrm{~min}$ at $37^{\circ} \mathrm{C}$ in the wet box, washed three times with PBS and the incubated with $200 \mu \mathrm{l}$ DAB (diaminobenzidine) for $10 \mathrm{~min}$ at room temperature. Until the background of slices became brown, the reactions were stopped and washed DAB with deionized water for several times.

\subsection{Stereological Analysis}

Slices stained with HE were taken photos with the image acquisition system (Olympus IX71, Japan). The whole tubules area, lumen area and cellular thickness were measured. The mean values of every measured morphological parameter, the rate of lumen area to whole area and the rate of cellular thickness to whole area were calculated. Ultimately, the parameters were statistically analyzed between the vasectomy group and the sham operated group.

\subsection{Statistical Analysis}

All data were analyzed with SPSS (v 13.0; Chicago, IL,
USA) using Independent-Samples Student's test. p < 0.05 was considered statistically significance. Data in the table are shown as mean \pm SEM (standard of error of the mean).

\section{Results}

\subsection{Effects of Vasectomy on Morphology Changes}

The histological changes under the light microscopy in the testis, epididymis and vas deferens after vasectomy were compared and shown in Figure 1.

The germinal epithelium did not show any sign of damages, and the arrangements, counts of spermatogenesis was not altered substantially in any animal of the sham operated group (Figure 1(a)). While in the vasectomy group, seminiferous epithelium became thinned and loose, cells were in disorder. The seminiferous tubules were expanded. Spermatids and spermatozoa could not be observed clearly in the seminiferous tubules (Figure 1(b)). In the sham operated group, the cilium in the wall of the epididymis duct was arranged orderly, pseudostratified columnar epithelial cells arranged in neat rows without slightest deformation (Figure 1(c)). However, the epididymis was significantly distended and enlarged in the vasectomy group. The pseudostratified columnar epithelial cells were compressed and deformed. There were plenty of fragmentations deposited in the epididymis lumen (Figure 1(d)).

In the sham operated group, the epithelium cells of the vas and the muscular layers were both intact (Figure 1(e)). While in the vasectomy group, the epithelium was compressed and deformed, and at the basement, fibroplasias could be observed (Figure 1(f)).

\subsection{Effect of Vasectomy on the Cell Apoptosis}

The germinal epithelial cells of testis of the sham operated group did not go apoptosis. The spermatogenic cells at all levels existed and arranged in neat rows. In the testis of the sham-operation group, only $4 \%$ of spermatogenic cells underwent apoptosis among 500 cells (Figure 2(a)). In contrast, only a few normal cells could be observed in the vasectomy group. About $89 \%$ of the spermatogenic cells in the vasectomy group underwent apoptosis, including most spermatogonia, spermatocytes, spermatids and sperm (Figure 2(b)).

Only small amount cells about $16 \%$ of epididymis epithelial cells in the sham operated group went apoptosis, which might be the normal programmed cell death (Figure 2(c)). While the cells were severed significantly apoptosis after the vas deferens obligated for 12 months. Almost all the cells were stained brown which indicated the DNA of these cells broken (Figure 2(d)). The apoptosis index in the vasectomy group was $96 \%$. 

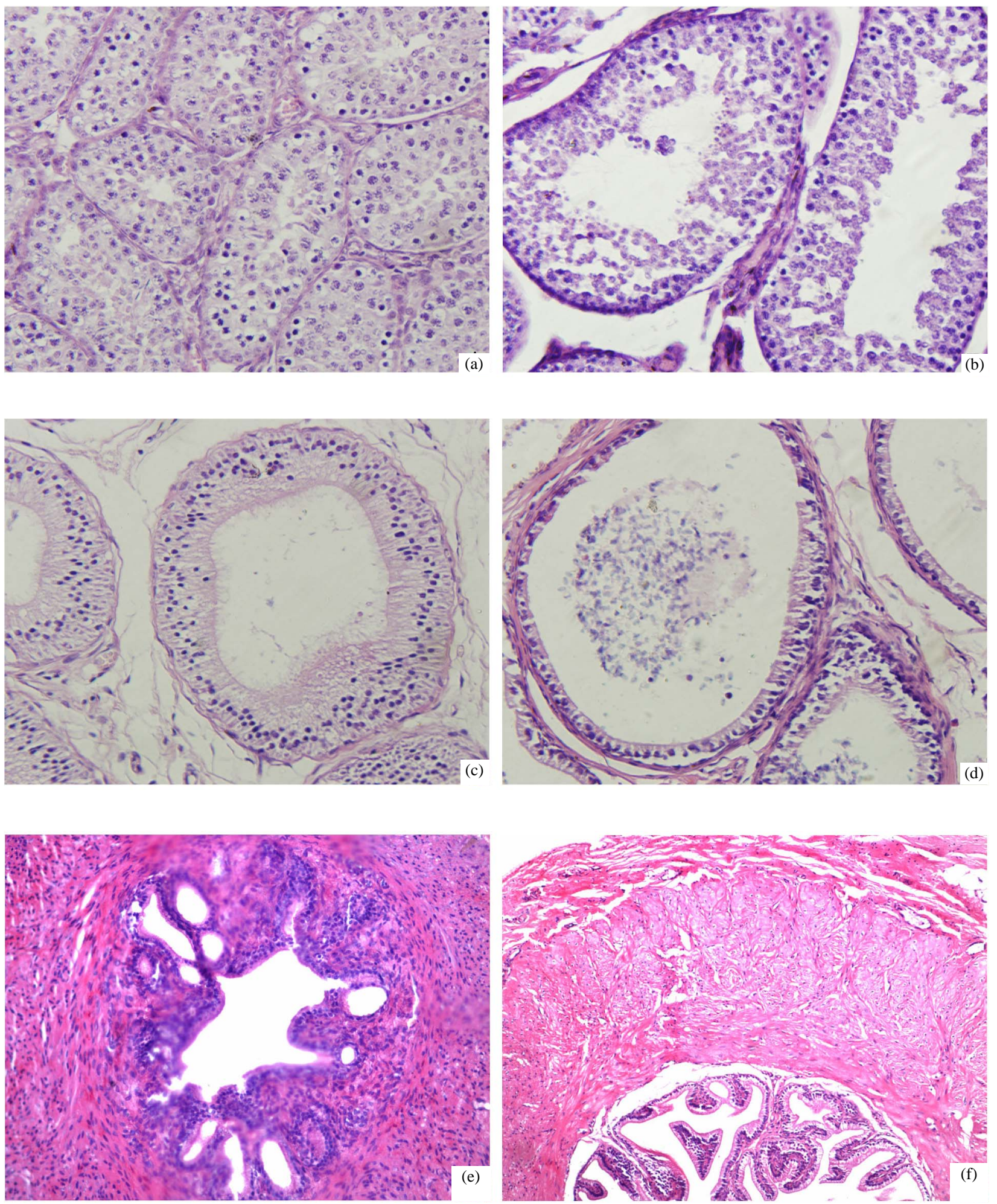

Sections were prepared and stained with H \& E. In the sham group, the spermatogenic cells of testicular seminiferous tubules arranged in neat rows and no significant changes were seen (a); Spermatogenic cells of the seminiferous tubules became loosen and irregularly arranged in the vasectomy group (b). Epithelial cells in epididymis of the sham operated group arranged in neat rows, cilia can be seen clearly (c); The epididymis of the vasectomy group was expanded with epithelium compressed. A lot of fragments deposited and cilia were disappeared (d); The epithelial cells of vas deferens in the sham operated group were arranged orderly, no cell deformed (e); Mucosal folds of the vas deferens lumen in the vasectomy group became shallower or even disappear, muscular fibrosis can be seen (f). a, b, c, d × 400, e, $\mathrm{f} \times 200$.

Figure 1. Histological changes of the testis, epididymis and vas deferens of two groups. 

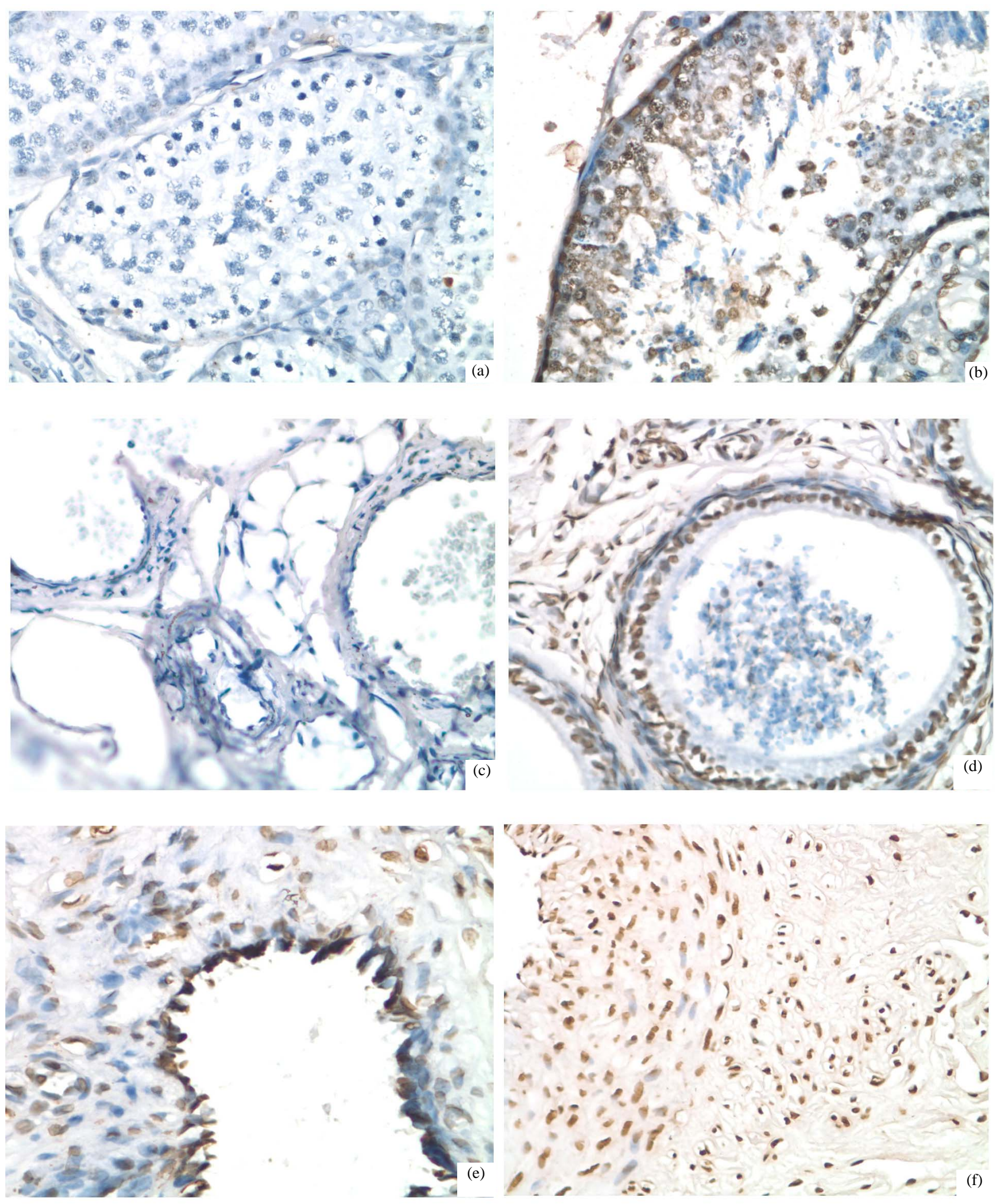

In the sham operated group, spermatogenic cells at all levels existed and were arranged densely in neat rows, cells went apoptosis can be seen occasionally (a); Spermatogonia, spermatocytes, spermatids in the vasectomy group went apoptosis (nucleus brown stained) (b); Only a small amount of cell went apoptosis in the epididymis of the sham operated group (c); Most of the nucleus of the epitheliums in the epididymis was brown stained in the vasectomy group (d); No differences of cell apoptosis were observed between the two groups (sham operated and vasectomy group, e, f). a, b, c, d, e, f $\times 400$.

Figure 2. Cell apoptosis of tissues of two groups. 
By careful observation of the epithelium and muscular cells in both groups, brown staining of nucleus was not clear in both groups. Most of the brown staining observed in the plasma of the cells. So we think there were not significant differences of cell apoptosis between the two groups. (Figure 2(f)).

\subsection{Stereological Analysis Results}

The area of spermatozoa/epididymis tubules, the area of lumen, the thickness of cellular, the rate of lumen area to whole area and the rate of cellular thickness to whole area were shown in Table 1. Most of the items measured shown significant differences $(\mathrm{P}<0.05)$. Compared with the sham group, the whole area of epididymis and lumen areas of tubules of the vasectomy group were great increased. The cellular thickness of epididymis became thinner. Although the celluar layer thickness of testis didn't show the significance between the sham group, the rate of cellular thickness to whole area shown significant difference $(P=0.0054)$. These changes may be caused by the continuous increasing pressure for long term.

\section{Discussion}

The observed morphologic and stereological changes induced by vasectomy in rabbits in our study demonstrated an obvious damage to the reproductive tissues for 12 months. Increasing pressure in the vas deferens may destroy the normal structure of the seminiferous epithelium and influence the function of spermatogenesis. The effects of vasectomy on epididymis may also influence the capacitation or maturation of sperms.

Urry et al [7]. found that vasectomy could influence spermatogenesis in adult dogs. Jarow et al [8]. reported that severe histological changes could be observed at post- vasectomy testicular tissues and autoimmune response enhancement was also verified. These results are consistent with our experiments.

A large number of studies performed on different species in order to find the reasons of these damages. Until now, the etiologies of reproductive damages after vasectomy were still not very clear. They may be mechanical, hormonal, immunologic, or any combination of these or other factors.

After vasectomy, the continuous production of sperm which could not be drained, increase the pressure of the seminiferous tubules. The inner pressure of testis, epididymis and vas deferens significantly increased and cause the expansion of epididymis and vas deferens [9]. Vare suggested that the changes of the seminiferous tubules probably result from blood stasis, which, is caused by increased internal pressurec [10].

Furthermore, when vasectomy was done, the sperm exposed and the exposure sperm were phagocytized by phagocytes and the epididymis epithelium, then stimulate their own immune system and promote the formation of anti-sperm antibodies [11]. At the same time, the sperm cannot be eliminated from the body after vasectomy, the sperm become death and they contribute to the produce of anti-sperm antibodies. The sperm antibodies can also promote the occurrence of sperm specific immune response [12]. Some researchers have reported that the percenttage of anti-sperm antibodies after vasectomy formed was in $60 \%-80 \%$ [13]. Many studies have shown that antisperm antibodies have a relationship with the changes of testicular morphology. Chehval et al [14] found that antisperm antibody levels were significantly increased after unilateral vasectomy in adult rats. Hence, leading to changes of testicular structure or infer that anti-sperm antibodies may damage the testicular structure.

Table 1. Stereological analysis results of two groups male rabbits after twelve months.

\begin{tabular}{|c|c|c|c|}
\hline Items & Sham group ( $\bar{X} \pm S D$ ) & Vasectomy group ( $\bar{X} \pm S D$ ) & $P$ value \\
\hline \multicolumn{4}{|c|}{ Whole area $\left(\mu \mathrm{m}^{2}\right)$} \\
\hline Testis & $16847.80 \pm 2400.85$ & $46122.69 \pm 6205.24$ & 0.0901 \\
\hline Epididymis & $55750.71 \pm 8412.33$ & $129420.05 \pm 17716.90$ & 0.0451 \\
\hline \multicolumn{4}{|c|}{ Lacunar area $\left(\mu \mathrm{m}^{2}\right)$} \\
\hline Testis & $462.54 \pm 143.23$ & $24257.89 \pm 5804.83$ & 0.0021 \\
\hline Epididymis & $15479.01 \pm 469.31$ & $111747.79 \pm 11590.90$ & 0.0039 \\
\hline \multicolumn{4}{|c|}{ Lacunar area/Whoel area } \\
\hline Testis & $0.0274 \pm 0.0068$ & $0.5255 \pm 0.1034$ & 0.0069 \\
\hline Epididymis & $0.2829 \pm 0.0438$ & $0.8685 \pm 0.0699$ & 0.5609 \\
\hline \multicolumn{4}{|c|}{ Cellular layer thickness $(\mu \mathrm{m})$} \\
\hline Testis & $66.4600 \pm 11.8958$ & $23.0580 \pm 2.9048$ & 0.1356 \\
\hline Epididymis & $59.0938 \pm 6.9416$ & $11.1390 \pm 0.9479$ & 0.0006 \\
\hline \multicolumn{4}{|c|}{ Cellular layer thickness/Whole area $(1 / \mu \mathrm{m})$} \\
\hline Testis & $0.0039 \pm 0.0002$ & $0.0005 \pm 0.0001$ & 0.0054 \\
\hline Epididymis & $0.0011 \pm 0.0002$ & $0.0001 \pm 0.00001$ & 0.0084 \\
\hline
\end{tabular}


The production of reactive oxygen species (ROS) after vasectomy might be another reason for these damages. After vas deferens ligated, the sperm deposition could cause local inflammatory response and generate ROS. ROS generation may have a certain relationship with the testicular tissue damage [15]. Excessive ROS products may also through the peroxidation of sperm membrane unsaturated fatty acids to cause sperm dysfunction [16]. Some reports suggested that the changes of reproductive hormones level might be associated with these damages [17-19].

Other reports have also confirmed that the level of reproductive hormones in rats changed after vasectomy [19], the pressure of the vas deferens increased [9], the antisperm antibodies produced [11]. All these can promote the expression of gene p53 and Bax [20]. P53 along with Bax and these damages may be the main reasons of the degree of apoptosis increasing.

Vasectomy has been generally considered as a safe and effective male contraception producer. However, longterm blockage of the vas deferens may cause damages to reproductive organs along with the time increasing. As shown in our results, the most direct consequences of this damage were led to the decline in male fertility and also can cause the produce of anti-sperm antibodies. The antisperm antibodies can exist in 20 - 30 years in some patients and this can cause the autoimmune infertility after vasectomy reversal [21].

In the research of Mcvicar et al., patients who had undergone a vasectomy more than 5 years were examined, and the clinical pregnancy rate was significant reduced [1]. Furthermore, the epididymis plays a crucial role in the storage and maturation of spermatozoa produced by the testis [22]. After the vasectomy, the normal histology of the epididymis was damaged. Thus the function of the epididymis might be affected. The spermatozoa could not maturate and then affect the recovery rate of pregnant after reversal.

Meanwhile, the requirements of vasectomy reversal increase every year, more than $6 \%$ of men who undergo vasectomy will request reversal [6]. Although the continuous progress with the microsurgery, the success rate of vas deferens can rise up to $96 \%$, but the recovery rate of pregnant is only $57 \%$ [23].

As far as concerned, the safety of this procedure needs more attentions. Our findings infer that longer term vasectomy might influence fertility recovery in an irreversible way.

\section{Conclusion}

In summary, the dangers of long term vasectomy should not be ignored, the vas deferens targeting contraception technology need to be focused on none-complete-blocking methods, such as one-end-opened vasectomy or fil- tering intra-vas devices research and development.

\section{Acknowledgements}

This work was supported by the funds from the 11th FiveYear National technology R \& D Program of China (grant no.2006BAI03B03).

\section{REFERENCES}

[1] C. M. McVicar, D. A. O’Neill, N. McClure, B. Clements, S. McCullough and S. E. Lewis, "Effects of Vasectomy on Spermatogenesis and Fertility Outcome after Testicular Sperm Extraction Combined with ICSI," Human Reproduction, Vol. 20, No. 10, 2005, pp. 2795-2800.

[2] E. J. Vrijhof, A. de Bruine, A. A. Lycklama a Nijeholt and L. H. Koole, "A Polymeric Mini-Stent Designed to Facilitate the Vasectomy Reversal Operation: A Rabbit Model Study,” Biomaterials, Vol. 25, No. 4, 2004, pp. 729-734. doi:10.1016/S0142-9612(03)00569-6

[3] X. B. Huang, J. P. Suo, C. Y. Chen, Q. L. Du, J. Y. Shen and J. L. Zhou, "Initial Studies on a Novel Filtering-Type Intra-Vas Device in Male Dogs," Contraception, Vol. 81, No. 4, 2010, pp. 350-354.

doi:10.1016/j.contraception.2009.10.012

[4] C. J. Flickinger, J. C. Herr, S. S. Howards, D. Caloras, E. S. Yarbro, D. R. Spell, et al., "The Influence of Vasovasostomy on Testicular Alterations after Vasectomy in Lewis Rats,” Anatomical Record, Vol. 217, No. 2, 1987, pp. 137-145. doi:10.1002/ar.1092170205

[5] S. W. McDonald and R. J. Scothorne, "A Quantitative Study of the Effects of Vasectomy on Spermatogenesis in Rats,” Journal of Anatomical, Vol. 159, 1988, pp. 219225.

[6] M. Goldstein, "Vasectomy Reversal," Comprehensive Therapy, Vol. 19, No. 1, 1993, pp. 37-41.

[7] R. L. Urry, K. A. Dougherty and A. T. Cockett, "Vasectomy and Vasovasostomy I. Timing of Histologic changes in Immature and Mature Dog Testis after Vasectomy," Fertility and Sterility, Vol. 27, No. 8, 1976, pp. 937-944.

[8] J. P. Jarow, E. T. Goluboff, T. S. Chang and F. F. Marshall, "Relationship between Antisperm Antibodies and Testicular Histologic Changes in Humans after Vasectomy,” Urology, Vol. 43, No. 4, 1994, pp. 521-524. doi:10.1016/0090-4295(94)90246-1

[9] C. J. Flickinger, J. C. Herr, M. L. Baran and S. S. Howards, "Testicular Development and the Formation of Spermatic Granulomas of the Epididymis after Obstruction of the Vas Deferens in Immature Rats," Journal of Urology, Vol. 154, No. 4, 1995, pp. 1539-1544. doi:10.1016/S0022-5347(01)66924-6

[10] A. M. Vare and P. C. Bansal, "The Effects of Ligation of Cauda Epididymidis on the Dog Testis," Fertility and Sterility, Vol. 25, No. 3, 1974, pp. 256-260.

[11] B. C. Fauser, A. B. Galway and A. J. Hsueh, "Inhibitory Actions of Interleukin-1 Beta on Steroidogenesis in Primary Cultures of Neonatal Rat Testicular Cells” Acta 
Endocrinology, Vol. 120, No. 4, 1989, pp. 401-408.

[12] H. H. Handley, C. J. Flickinger and J. C. Herr, Post-Vasectomy Sperm Autoimmunogens in the Lewis Rat,” Biology Reproduction, Vol. 39, No. 5, 1988, pp. 1239-1250. doi:10.1095/biolreprod39.5.1239

[13] R. L. Urry, J. Thompson and A. T. Cockett, "Vasectomy and Vasovasostomy II. A Comparison of Two Methods of Vasovasostomy: Silastic Versus Chromic Stents,” Fertility and Sterility, Vol. 27, No. 8, 1976, pp. 945-950.

[14] M. J. Chehval, R. Doshi, C. F. Kidd, T. Winkelmann and V. Chehval, “Antisperm Autoantibody Response after Unilateral Vas Deferens Ligation in Rats: When Does it Develop? Journal of Andrology, Vol. 23, No. 5, 2002, pp. 669-673.

[15] K. Aydos, B. Kupeli, T. Soygur, A. Unsal, E. Erden, O. Tulunay, et al., "Analysis of the Relationship between Histologic Alterations and the Generation of Reactive Oxygen Species in Vasectomized Rat Testes," Urology, Vol. 51, No. 3, 1998, pp. 510-515. doi:10.1016/S0090-4295(97)00718-8

[16] R. J. Aitken, J. S. Clarkson, T. B. Hargreave, D. S. Irvine and F. C. Wu, "Analysis of the Relationship between Defective Sperm Function and the Generation of Reactive Oxygen Species in Cases of Oligozoospermia,” Journal of Andrology, Vol. 10, No. 3, 1989, pp. 214-220.

[17] Z. N. Mo, X. Huang, S. C. Zhang and J. R. Yang, "Early and Late Long-Term Effects of Vasectomy on Serum Testosterone, Dihydrotestosterone, Luteinizing Hormone and Follicle-Stimulating Hormone Levels," Journal of Urology, Vol. 154, No. 6, 1995, pp. 2065-2069.
doi:10.1016/S0022-5347(01)66696-5

[18] K. D. Smith, R. K. Tcholakian, M. Chowdhury and E. Steinberger, "An Investigation of Plasma Hormone Levels before and after Vasectomy," Fertility and Sterility, Vol. 27, No. 2, 1976, pp. 144-151.

[19] B. Geierhaas, S. R. Bornstein, H. Jarry, W. A. Scherbaum, M. Herrmann and E. F. Pfeiffer, "Morphological and Hormonal Changes Following Vasectomy in Rats, Suggesting a Functional Role for Leydig-Cell Associated Macrophages," Hormone Metabolism Research, Vol. 23, No. 8, 1991, pp. 373-378. doi:10.1055/s-2007-1003704

[20] K. Shiraishi, K. Naito and K. Yoshida, "Vasectomy Impairs Spermatogenesis through Germ Cell Apoptosis Mediated by the p53-Bax Pathway in Rats," Journal of Urology, Vol. 166, No. 4, 2001, pp. 1565-1571. doi:10.1016/S0022-5347(05)65831-4

[21] D. A. Ohl and R. K. Naz, "Infertility Due to Antisperm Antibodies,” Urology, Vol. 46, No. 4, 1995, pp. 591-602. doi:10.1016/S0090-4295(99)80282-9

[22] S. Filippi, G. B. Vannelli, S. Granchi, M. Luconi, C. Crescioli, R. Mancina, et al., "Identification, Localization and Functional Activity of Oxytocin Receptors in Epididymis," Molecular Cell Endocrinology, Vol. 193, No. 1-2, 2002, pp. 89-100. doi:10.1016/S0303-7207(02)00101-6

[23] C. G. Schrepferman, M. R. Carson, A. E. Sparks and J. I. Sandlow, "Need for Sperm Retrieval and Cryopreservation at Vasectomy Reversal,” Journal of Urology, Vol. 166, No. 5, 2001, pp. 1787-1789. doi:10.1016/S0022-5347(05)65676-5 